\title{
Visual tracking in very preterm infants at 4 mo predicts neurodevelopment at 3 y of age
}

\author{
Ylva Fredriksson Kaul', Kerstin Rosander ${ }^{2}$, Claes von Hofsten ${ }^{2}$, Katarina Strand Brodd ${ }^{1,3}$, Gerd Holmström ${ }^{4}$, Alexander Kaul' , \\ Birgitta Böhm ${ }^{5}$ and Lena Hellström-Westas ${ }^{1}$
}

BACKGROUND: Typically developing infants track moving objects with eye and head movements in a smooth and predictive way at 4 mo of age, but this ability is delayed in very preterm infants. We hypothesized that visual tracking ability in very preterm infants predicts later neurodevelopment.

METHOD: In 67 very preterm infants (gestational age $<32 \mathrm{wk}$ ), eye and head movements were assessed at 4 mo corrected age while the infant tracked a moving object. Gaze gain, smooth pursuit, head movements, and timing of gaze relative the object were analyzed off line. Results of the five subscales included in the Bayley Scales of Infant Development (BSID-III) at $3 y$ of age were evaluated in relation to the visual tracking data and to perinatal risk factors.

RESULTS: Significant correlations were obtained between gaze gain and cognition, receptive and expressive language, and fine motor function, respectively, also after controlling for gestational age, severe brain damage, retinopathy of prematurity, and bronchopulmonary dysplasia.

CONCLUSION: This is the first study demonstrating that the basic ability to visually track a moving object at 4 mo robustly predicts neurodevelopment at $3 \mathrm{y}$ of age in children born very preterm.

C hildren born very preterm, i.e., with a gestational age $<32 \mathrm{wk}$, are at increased risk for neurodevelopmental impairments $(1,2)$. In particular, these children show deficits in visuospatial perceptual abilities, which are not explained by their general intelligence level (3-5). Cognitive and neurodevelopmental difficulties in very preterm infants can often be predicted already in the neonatal period, e.g., by presence of gray and white matter abnormalities on magnetic resonance imaging at term equivalent age. However, not all infants with brain injury will have a poor outcome, and also infants with no obvious neonatal brain injury may have an adverse neurodevelopment $(4,6)$.

Visual tracking is one of the earliest developing cognitive abilities which are present already soon after birth. Unless the object is large and moving very slowly, newborn infants predominantly use saccades. During the second month after birth, the ability to stabilize gaze on a moving object through smooth pursuit eye movements improves greatly (7) and tracking is achieved by a combination of head and eye movements $(7,8)$. In normally developing infants, horizontal smooth pursuit approaches adult levels around 4 mo of age (8). The contribution of the head increases and at 5 mo infants use the head to follow an object more than adults $(7,9)$. Visual tracking performance has been investigated in a number of clinical conditions in childhood and later, and is associated with cognitive functions as well as with psychiatric disorders $(10,11)$.

In a population-based study of very preterm infants, we used a method of quantitative measurement of eye and head movements in relation to a horizontally oscillating object developed by von Hofsten and Rosander $(7,9)$. The study protocol at $4 \mathrm{mo}$ included evaluation of "smooth pursuit gain", i.e., the ability to keep the eyes focused on a moving object and to follow it smoothly, and "gaze gain" which refers to the combination of visual tracking through smooth pursuit, head movements, and saccades. To stabilize gaze on the object, eye and head movements need to be coordinated in time and space. In this study, gain is a spatial parameter, which refers to the ratio between gaze, smooth pursuit or head movement amplitudes and the object motion amplitude. Gaze and smooth pursuit should optimally be stabilized on the object which gives a ratio of 1 , a ratio less than or larger than 1 indicates that tracking amplitude was smaller (undershooting) or greater than the object amplitude (overshooting) wich are both detrimental to tracking. Thus, "smooth pursuit gain", "head gain", and "gaze gain" describes how close smooth pursuit, head or gaze, respectively, anticipated the position of the object. The temporal parameter "timing of gaze" described whether the gaze was lagging or leading the object. To align gaze with the object motion, prediction of the objects direction and velocity is required which also includes prediction of the sinusoidal motion.

We have previously demonstrated that very preterm infants in the present study population had a delayed development of 


\section{Articles | Kauletal.}

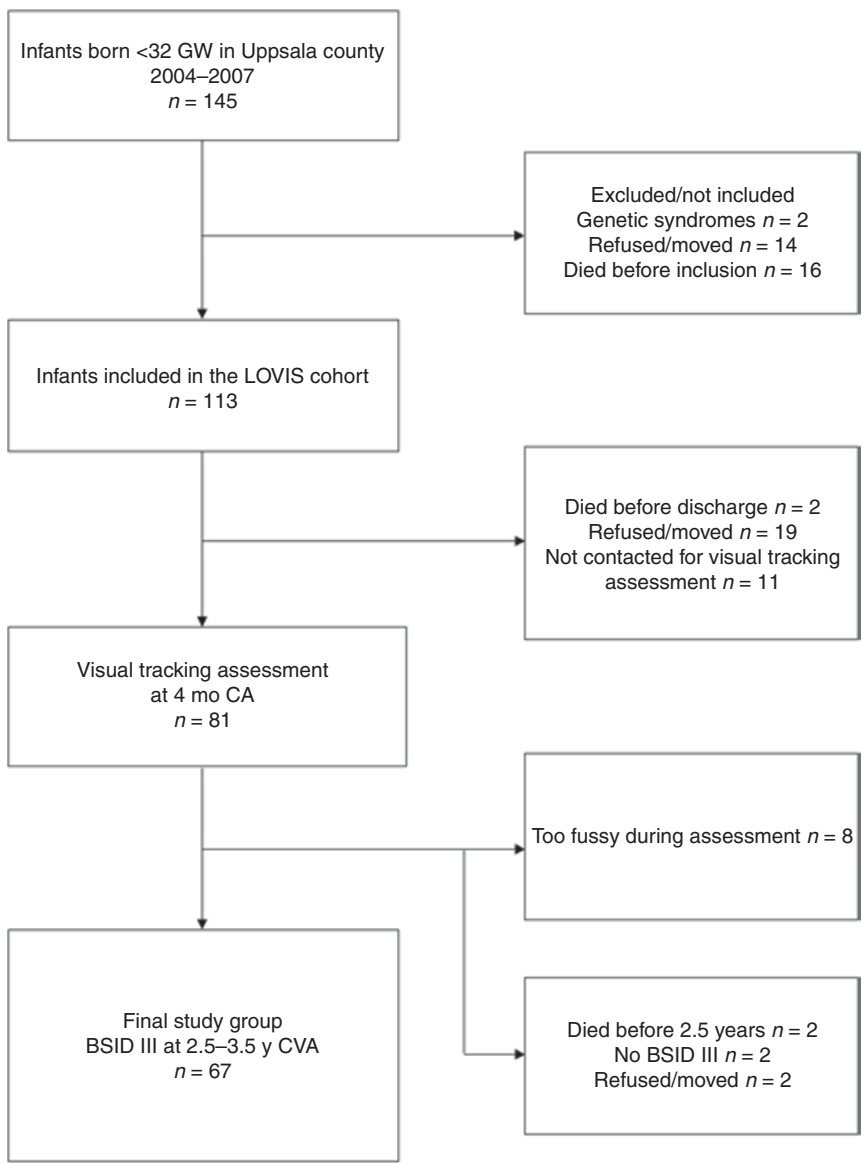

Figure 1. Flow chart of the study group.

visual tracking at 2 and 4 mo corrected age (12). Several neonatal risk factors correlated with the delayed visual tracking ability, but only presence of periventricular leukomalacia was independently associated with the visual tracking performance (13). In the present study, we hypothesized that visual tracking ability at 4 mo corrected age is an early indicator of neurobehavioral function and correlates with later neurodevelopment, assessed by the Bayley Scales of Infant Development (BSID version III) at $3 \mathrm{y}$ of age.

\section{RESULTS}

Patient characteristics ar given in Figure 1 and Table 1. Means and SDs for tracking parameters and BSID III subscale scores are given in Table 2. Figure 2a-d gives examples of visual tracking patterns in two infants.

\section{Gain}

Gaze gain explained a large part (22-32\%) of the variance in the BSID III cognitive, receptive language, expressive language, and fine motor scores (cognition: $R^{2}=0.315, P<0.001$; receptive language: $R^{2}=0.224, P=0.001$; expressive language: $R^{2}=0.285$, $P<0.001$; fine motor: $\left.R^{2}=0.218, P=0.001\right)$. An optimal tracking of the object, with the gaze following the complete trajectory of the target without overshoot at the turning points (Figure 3a), was associated with higher scores on all four BSID III subscales.
Table 1. Characteristics of the study population

\begin{tabular}{llc}
\hline & & $\begin{array}{c}\text { Study population, } \\
N=67\end{array}$ \\
\hline Perinatal data & Antenatal steroids, $n$ (\%) & $46(69 \%)$ \\
& Cesarean section, $n$ (\%) & $41(61 \%)$ \\
& Gestational age, weeks & $28.3(2.6)$ \\
Neonatal risk & Birth weight, g & $1,162(363)$ \\
factors & Severe brain injury (IVH 3-4 and/or & $4(6 \%)$ \\
& PVL), $n$ (\%) & \\
& Retinopathy of prematurity any & $24(36 \%)$ \\
& degree, $n$ (\%) & \\
& Retinopathy of prematurity $\geq 3, n(\%)$ & $8(12 \%)$ \\
& Bronchopulmonary dysplasia, $n(\%)$ & $16(24 \%)$ \\
\hline
\end{tabular}

Regional cohort of 67 very preterm infants who underwent visual tracking assessment at 4 mo corrected age. Data are mean values (SDs) and numbers (percentages), respectively.

aNeonatal risk factors in the regression analyses also include gestational age. IVH, intraventricular hemorrhage; PVL, periventricular leukomalacia.

Table 2. Visual tracking parameters at 4 mo corrected age and outcome at $3 \mathrm{y}$ in a cohort of very preterm infants

\begin{tabular}{llc}
\hline & & $\begin{array}{c}\text { Study population, } \\
N=67\end{array}$ \\
\hline Tracking parameter & Gaze gain & $0.90(0.26)$ \\
& Smooth pursuit gain & $0.44(.021)$ \\
& Head gain & $0.28(0.20)$ \\
& Timing (s) & $-0.16(0.17)$ \\
Three-year outcome & Cerebral palsy, $n(\%)$ & $5(7 \%)$ \\
& Autism, $n(\%)$ & $5(7 \%)$ \\
& Strabismus or major & $12(18 \%)$ \\
& refractive error, $n$ (\%) & \\
& Visual impairment, $n(\%)$ & $0(0 \%)$ \\
& BSID III Cognitive score & $9.9(2.3)$ \\
& BSID III Receptive language & $10.3(2.9)$ \\
& Score & \\
& BSID III Expressive language & $10.9(3.4)$ \\
& Score & \\
BSID III Fine motor score & $11.3(3.4)$ \\
BSID III Gross motor score & $8.0(2.6)$
\end{tabular}

The table shows ratios for gaze, smooth pursuit, and head gain (optimal is 1) as well as for timing (s) and outcome including BSID III subscale scores. Data are mean values (SDs) and numbers (percentages), respectively.

BSID-III, Bayley Scales of Infant and Toddler Development version III.

Smooth pursuit gain was also significantly associated with the cognitive and expressive language subscales, but explained only around $7 \%$ of the variance (cognition: $R^{2}=0.069$, $P=0.036$; expressive language: $R^{2}=0.067, P=0.044$ ). The larger the proportion of the object trajectory that was followed by smooth pursuit eye movements, the higher were the scores in these subscales (Figure $3 b$ ). Head gain was significantly associated with the cognitive, expressive language, and fine motor subscales, explaining $7-11 \%$ of variance (cognition: $R^{2}=0.092, P=0.019$; expressive language: $R^{2}=0.071, P=$ 0.039 ; fine motor: $R^{2}=0.108, P=0.010$ ). These data indicate 
a
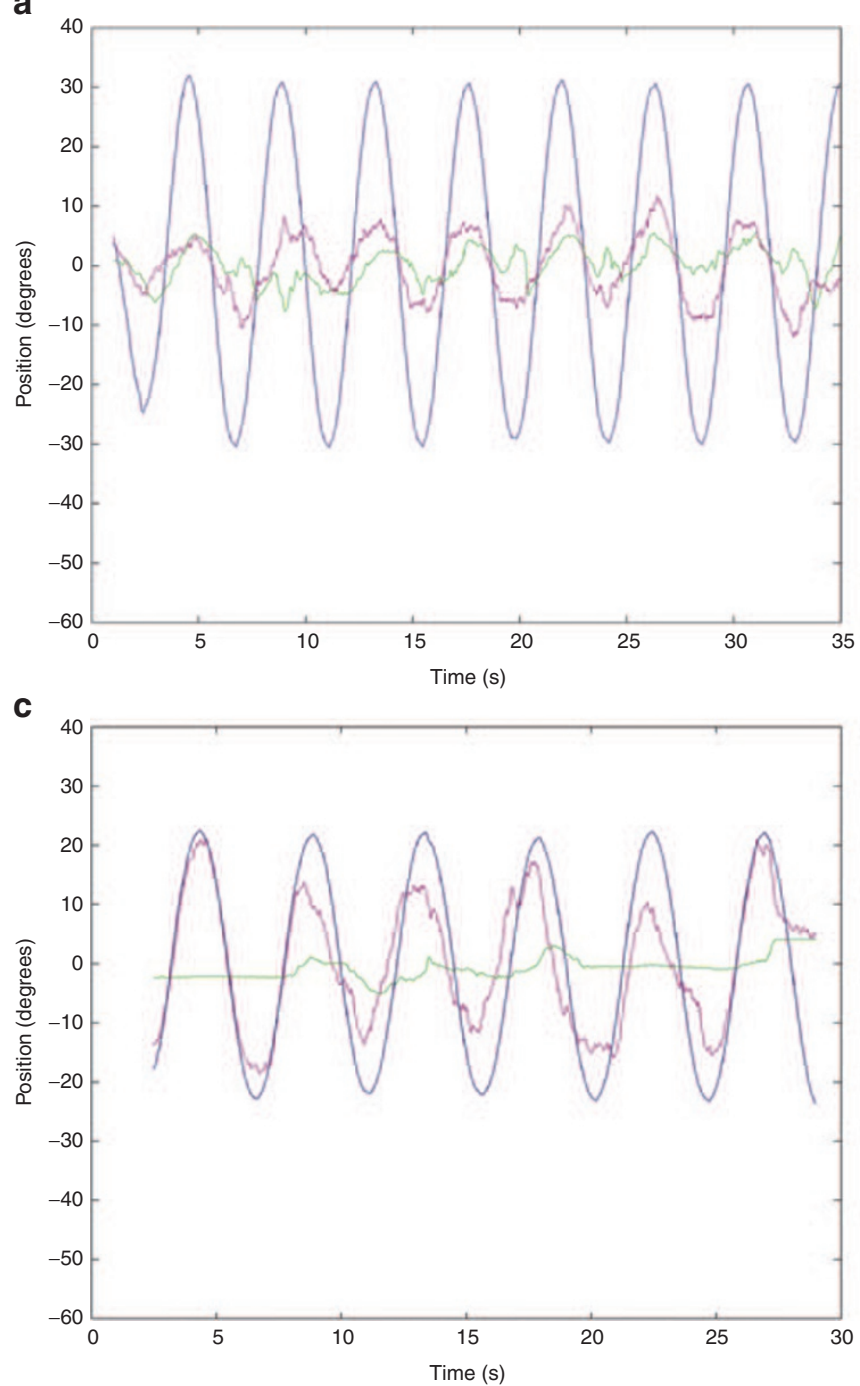

b

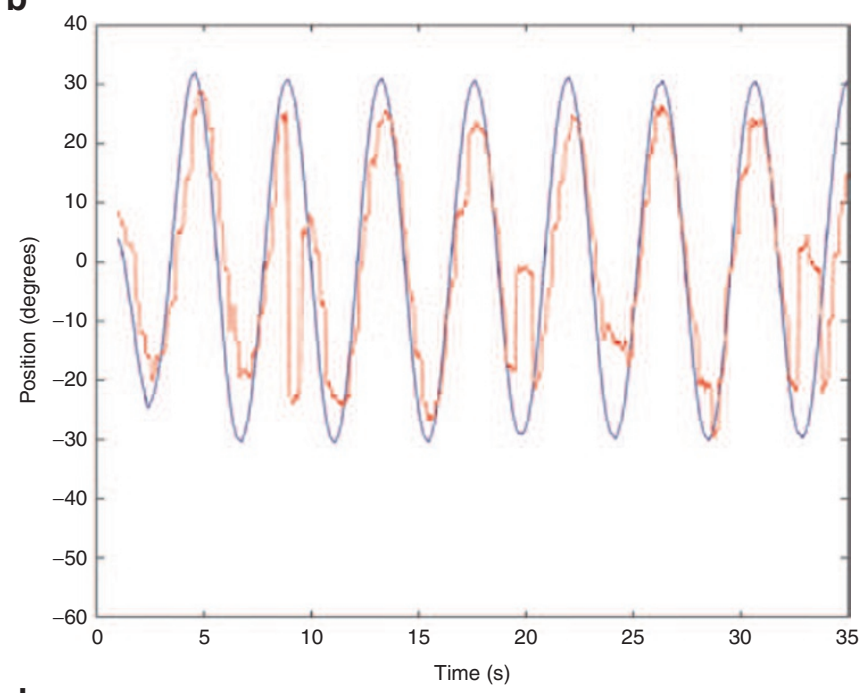

d

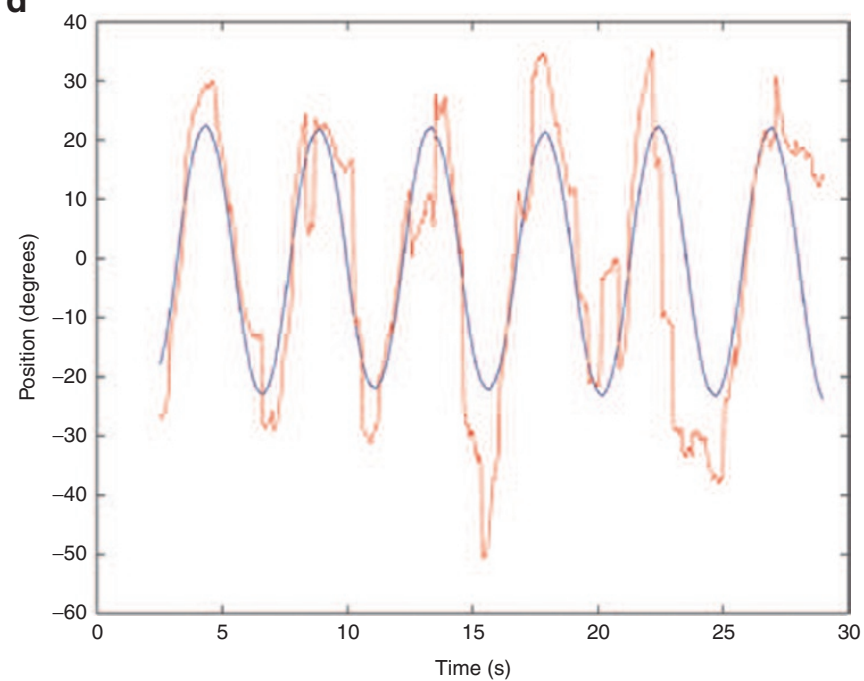

Figure 2. Examples of visual tracking recordings in two very preterm infants. Position of the smooth pursuit part of the eye movement (magenta), the head movement (green), the gaze (red), and the object (blue) are shown. (a,b) This infant, born at 26 gestational weeks, had gains 0.24 (smooth pursuit), 0.08 (head), and 0.65 (gaze), respectively. The number of saccades was $2.38 / \mathrm{s}$. The time difference (crosscorrelation gaze-object) was $-0.192 \mathrm{~s}$. In this subject, smooth pursuit and head movements varied in relation to the object and were both in and out of phase. (c,d) This infant, born at 31 gestational weeks, had gains 0.6 (smooth pursuit), 0.06 (head), and 1.16 (gaze), respectively. The number of saccades was 1.13/s. The time difference (crosscorrelation gaze-object) was $-0.025 \mathrm{~s}$. In this subject, gaze was overshooting in spite of moderate smooth pursuit and head movements. In both subjects, gaze gain gave the most accurate tracking, although the tracking parameters were coordinated differently to achieve that goal. At 4 mo corrected age, both infants were lagging the object slightly. At 3 y of age, both children had average results on all Bayley Scales of Infant Development (BSID) III subscales, except for the gross motor scale where they scored below average.

that using more head movements for tracking the object at 4 mo was beneficial for later development (Figure 3c).

\section{Timing}

At 4 mo corrected age, 63 of the 67 very preterm infants lagged the object motion. The timing of gaze in relation to the object was also important for neurodevelopment and explained $13-23 \%$ of the variances in the cognitive and language subscales (cognition: $R^{2}=0.155, P=0.002$; receptive language: $R^{2}=0.130, P=0.005$; and expressive language: $R^{2}=$ $0.225, P<0.001$ ) (Figure $3 \mathrm{~d}$ ).
No tracking parameter was associated with the BSID III gross motor subscale. The frequency of saccades was not related to the BSID III subscales.

\section{Neonatal Risk Factors}

Neonatal risk factors associated with the 3-y outcome and determined through univariate linear regressions were gestational age, degree of retinopathy of prematurity, and bronchopulmonary dysplasia, but not presence of severe brain injury (intraventricular hemorrhage 3-4/periventricular leukomalacia) (Table 3 ). 


\section{Articles $\mid$ Kauletal.}
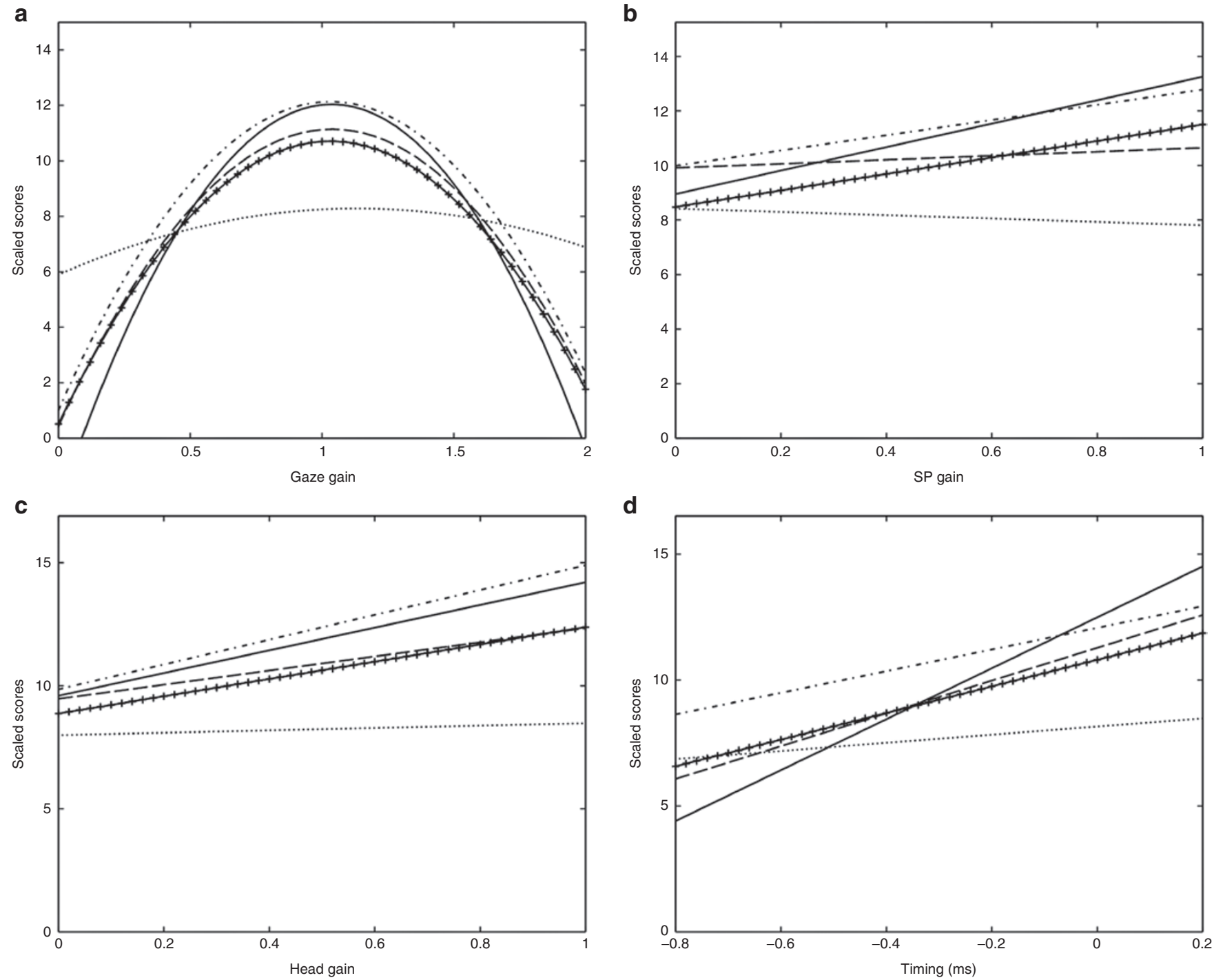

Figure 3. Regression lines for tracking parameters at 4 mo corrected age in relation to the Bayley Scales of Infant Development (BSID III) subscales at $3 y$ in a cohort of very preterm infants. Quadratic and linear regression lines for four tracking parameters (x-axis) in relation to the five BSID III subscales (y-axis): cognition ("railway" line); receptive language (dashed line); expressive language (solid line); fine motor (dash-dotted line); gross motor (dotted line). (a) Gaze gain: the curved line illustrates that infants with a perfect ratio between gaze tracking and object trajectory (i.e., 1) had the best outcome. Failure to follow the entire course of the object, or overshooting at the turning points, gives a ratio below or over 1 which is less favorable for outcome, as seen through the declining curve ends. (b) Regression lines for smooth pursuit gain, where 1 represents a perfect tracking through smooth pursuit. For cognition and expressive language the more of the object trajectory followed smooth pursuit eye movements, the better the outcome. As no infant had a smooth pursuit gain above 1, it was not possible to investigate if over shooting would be associated with lower BSID III results. (c) Regression lines for the relationships between head gain and 3-y outcome.The more of the object trajectory that was covered, the higher were the cognitive, expressive language and fine motor subscale scores. Again, there was no child with a ratio above 1. (d) Regression lines for timing and outcome were significant for the cognitive, receptive, and expressive language subscales. The $\mathrm{x}$-axis shows difference between gaze and object motion in milliseconds, with 0 for spot on tracking.

\section{Gaze Gain and Neonatal Risk Factors}

When the neonatal risk factors (gestational age, severe brain damage, retinopathy of prematurity, and bronchopulmonary dysplasia) were entered into a hierarchical regression model together with the visual tracking data, gaze gain explained unique variance not accounted for by the neonatal risk factors (Table 4). The increase in explanatory value by gaze gain $\left(\Delta R^{2}\right)$ was significant. Thus, the effect of gaze gain on neurodevelopmental scores was not the result of clinical risk factors influencing both early visual tracking ability and later development.
This was further confirmed as gestational age was entered as sole risk factor in a hierarchical regression model and the increase in explained variance $\left(\Delta R^{2}\right)$ was still significant for all four BSID III subscales (significance levels $(P)$ were found to be between 0.002 and 0.038 )

\section{DISCUSSION}

This is the first prospective study evaluating the relationship between early visual tracking and neurodevelopment. Our results demonstrate that integrated neural functional 
development at 4 mo corrected age, as reflected by visual tracking ability, is significantly related to later neurodevelopment in very preterm infants.

The most important result was that early visual tracking ability predicted cognitive, language, and fine motor development in these children. Gaze gain was the tracking parameter closest associated with the BSID III subscales. Smooth pursuit gain, head gain, and timing of gaze to object motion was related to several BSID subscales. In the regression analysis, gaze gain increased the predictive ability of neonatal risk factors for cognitive, receptive, and expressive language subscale scores, as well as for the fine motor subscale.

Keeping gaze fixed on a target requires sustained attention (11) since both temporal and spatial aspects of tracking have to be precise in order to stabilize the image on the retina $(7,14)$. Visual feedback is too slow to control this system and to generate synchronized behavior and therefore the velocity and direction of the object has to be predicted $(9,14,15)$. In contrast to typically developing infants, who usually demonstrate a lead of gaze relative to the object of 70 to $100 \mathrm{~ms}$ (16), a lead was present only in 4 of the 67 very preterm infants. However, the smaller the lag the better the developmental outcome, underlining the importance of adequate timing of gaze to object.

Successful tracking depends on the ability to coordinate saccades, smooth pursuit, and head movements into precise gaze gain and timing. Smooth visual tracking relies on smooth pursuit as well as on head movements (17). If smooth pursuit is poorly developed, head movements can compensate for this at moderate target velocities. It is therefore important not to restrain the movements of the head in the assessment, as this might compromise the gaze tracking.

Visual identification of objects and actions is important for the capability to communicate around them, and thus development of language accompanies that of visual processing (18). Our results are in line with those of Ellis et al. (19) who found that the ability to anticipate a visual sequence at 6 mo predicted receptive and expressive vocabulary at $2 \mathrm{y}$.

Fine motor function is dependent on eye-hand coordination, which matures early in life; at $4 \mathrm{mo}$, the child reaches successfully for both moving and stationary objects (20). When reaching, the child prepares to grasp the object by predictive timing of opening and closing of the hand (21). These adjustments are visually guided and it was therefore expected that early visual skills should be closely related to fine motor abilities.

The gross motor subscale showed no significant relationship with early visual tracking ability. The visual mechanisms required for gross motor development may differ from those underlying cognitive and language functions. Another possibility is that the BSID III gross motor subscale relies less on visual tracking abilities since no moving objects are included among the tasks at this age level. In the Swedish EXPRESS study, a national population-based study of infants born before 27 gestational weeks, the gross motor subscale identified fewer children with disability at $2.5 \mathrm{y}$ corrected age (prevalence $7 \%$ ) than the other BSID III subscales (prevalence 11-15\%) (22).
Table 3. Regressions between neonatal risk factors and neurodevelopmental outcome

\begin{tabular}{|c|c|c|c|c|c|c|c|c|}
\hline \multirow[b]{2}{*}{ BSID III } & \multicolumn{2}{|c|}{$\begin{array}{c}\text { Gestational } \\
\text { age }\end{array}$} & \multicolumn{2}{|c|}{$\begin{array}{c}\text { Retinopathy of } \\
\text { prematurity }\end{array}$} & \multicolumn{2}{|c|}{$\begin{array}{c}\text { Bronchopulmonar) } \\
\text { dysplasia }\end{array}$} & \multicolumn{2}{|c|}{$\begin{array}{c}\text { Severe brain } \\
\text { injury }\end{array}$} \\
\hline & $R^{2}$ & $P$ & $R^{2}$ & $P$ & $R^{2}$ & $P$ & $R^{2}$ & $P$ \\
\hline Cognition & 0.151 & 0.001 & 0.198 & 0.001 & 0.110 & 0.007 & 0.014 & 0.356 \\
\hline $\begin{array}{l}\text { Receptive } \\
\text { language }\end{array}$ & 0.057 & 0.056 & 0.089 & 0.016 & 0.066 & 0.039 & 0.004 & 0.631 \\
\hline $\begin{array}{l}\text { Expressive } \\
\text { language }\end{array}$ & 0.084 & 0.020 & 0.127 & 0.004 & 0.080 & 0.023 & 0.002 & 0.696 \\
\hline Fine motor & 0.094 & 0.013 & 0.091 & 0.015 & 0.148 & 0.002 & 0.000 & 0.993 \\
\hline Gross motor & 0.024 & 0.220 & 0.027 & 0.195 & 0.022 & 0.238 & 0.018 & 0.282 \\
\hline
\end{tabular}

Regressions between neonatal risk factors and Bayley Scales of Infant Development (BSID III) subscale scores at $3 y$ of age in a population of 67 very preterm infants. Summary of effect sizes $\left(R^{2}\right)$ and significance levels for univariate regressions. Presence of severe brain injury (IVH3-4/PVL) was not associated with outcome. Bold numbers indicate $P<0.05$.

Table 4. Relation between neonatal risk factors, gaze gain, and neurodevelopment at $3 y$

\begin{tabular}{lcccccccc}
\hline & \multicolumn{9}{c}{$\begin{array}{c}\text { Neonatal risk } \\
\text { factors }\end{array}$} & & \multicolumn{2}{c}{$\begin{array}{c}\text { Neonatal risk } \\
\text { factors and } \\
\text { gaze gain }\end{array}$} & & \multicolumn{2}{c}{ Difference } \\
\cline { 2 - 3 } BSID III & $R^{2}$ & $P$ & & $R^{2}$ & $P$ & & $\Delta R^{2}$ & $\Delta P$ \\
\hline Cognition & 0.271 & $\mathbf{0 . 0 0 2}$ & & 0.386 & $\mathbf{0 . 0 0 0}$ & & 0.114 & $\mathbf{0 . 0 1 0}$ \\
Receptive language & 0.130 & 0.105 & & 0.271 & $\mathbf{0 . 0 0 9}$ & & 0.140 & $\mathbf{0 . 0 1 0}$ \\
Expressive language & 0.179 & $\mathbf{0 . 0 2 8}$ & & 0.326 & $\mathbf{0 . 0 0 2}$ & & 0.147 & $\mathbf{0 . 0 0 6}$ \\
Fine motor & 0.195 & $\mathbf{0 . 0 1 8}$ & & 0.284 & $\mathbf{0 . 0 0 6}$ & & 0.089 & $\mathbf{0 . 0 4 7}$ \\
Gross motor & 0.055 & 0.536 & 0.056 & 0.797 & & 0.000 & 0.990 \\
\hline
\end{tabular}

Hierarchical regression models comparing explanatory value between a model of neonatal risk factors (gestational age, severe brain injury, retinopathy of prematurity, bronchopulmonary dysplasia) with a model of the same risk factors but also including gaze gain, and the difference $(\Delta)$ between the two models. $R^{2}$ is the proportion of the variance explained by each model and $\Delta R^{2}$ depicts how much more of outcome variance is explained through adding gaze gain to the model. Significance levels $(P)$ are given for the proportion of variance explained as well as for the increase in explanatory value $(\Delta P)$. Bold numbers indicate $P<0.05$.

It was also the only subscale in the present study that was not associated with clinical risk factors.

Visual attention and ability to follow moving targets, as assessed early in life through direct observation, has previously been shown to be associated with 12-mo outcome in preterm infants (23). In addition, cortical visual function measured with visual event-related potentials responses to visual fixational shifts at 3-7 mo corrected age predicted results on the Griffiths scale at $2 \mathrm{y}$ of age (3). Fixational shifts were delayed in very preterm infants without diagnosed brain damage and the authors suggested that evaluation of visual functioning might be a sensitive indicator of later subtle neurodevelopmental problems. Recently, neonatal visual fixation was demonstrated to predict visuomotor performance at $2 \mathrm{y}$, and visual reasoning at $5 \mathrm{y}$ of age (24).

Visual tracking ability relies on the function of the dorsal neural pathway, where information about spatial relationships, motion perception and control of motor actions are integrated with attention, motives, and comprehension $(3,25)$. At 4 mo of 


\section{Articles $\mid$ Kauletal.}

age, cortical processing of motion is still dominated by the direct pathway from retina to pulvinar and middle temporal area. For smooth pursuit, it is assumed that the frontal eye field is critical, as well as cerebellar areas via pontine nuclei. Subcortical nuclei (superior colliculus and substatia nigra) are also crucial for eye movements $(18,26)$. Lesions in these subcortical areas and basal ganglia give severe effects on eye movements (27). In addition, the prefrontal cortex is involved in predictive aspects of tracking (8). Retinopathy of prematurity and diffuse or localized white matter injury are prevalent in very preterm infants, and these conditions may affect the dorsal pathway and the maturation of both the pulvinar to middle temporal visual area and the primary visual pathways (26-28). It was shown in very preterm infants that reduced occipital cortical volumes at term equivalent age were associated with poor visual motion perception at 2 y of age (29). Abnormal visual tracking ability is present in many behavioral and psychiatric disorders, such as autism, attention deficit hyperactivity disorder, developmental coordination disorders, and dyslexia $(3,4,10,26,30)$, which also constitute a risk for children born very preterm. Visual tracking assessments and longitudinal follow-up studies may contribute to an increased understanding of the relation between neurodevelopmental profiles, and behavioral and psychiatric disorders in these children.

In the present study, only four infants were diagnosed with severe brain injury, and probably due to this low number, there were no significant associations between neonatal brain injury, visual tracking parameters, and 3-y outcome. A limitation was that no cerebral magnetic resonance images were performed in the neonatal period, and consequently mild to moderate white matter injury may not have been detected. However, cranial ultrasound examinations were performed by experienced pediatric radiologists at 35-36 gestational weeks and near term these examinations should be able to identify major white matter injury associated with adverse outcome $(31,32)$.

\section{Conclusions}

The present data demonstrates that visual tracking ability at 4 mo corrected age, and especially gaze gain, is an independent predictor of later neurodevelopment in very preterm infants, explaining around $30 \%$ of cognitive function and expressive language function at $3 \mathrm{y}$ of age. Gestational age was found to be an important and independent risk factor where low gestational age predicts low performance on BSID. Cognitive testing at school age could confirm the sustainability of the tracking results over time. Further studies are required to investigate whether measures of tracking ability should be included in the clinical routine for a better assessment of early neurological functioning. A visual tracking recording is clinically feasible, since it is quick to perform and not distressing for the infant and could be readily interpreted by a trained nurse or physician.

\section{METHODS}

\section{Subjects}

The LOngitudinal VISual follow-up of visuomotor development (LOVIS) study population included all very preterm infants in the County of Uppsala born at Uppsala University Hospital between January 2004 and December 2007 (12). A total of 145 very preterm

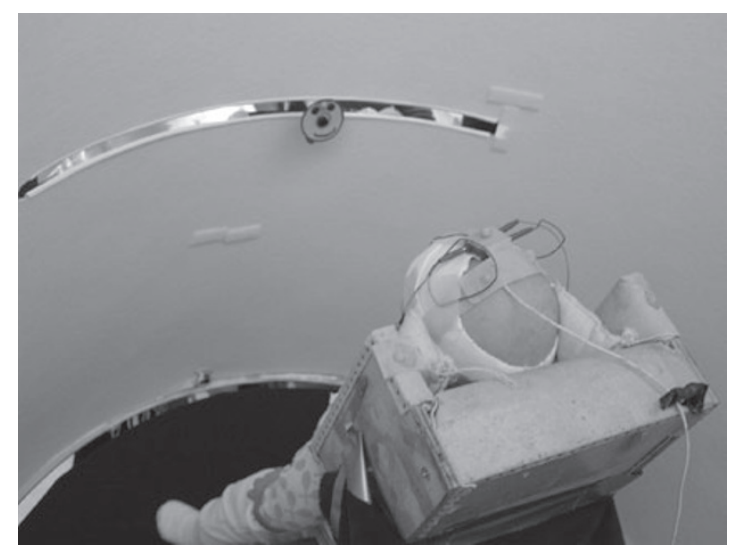

Figure 4. Experimental set up. Infant tracking the happy face inside the cylinder.

infants were eligible for the study; Figure 1 describes the steps for inclusion of the 67 infants who had visual tracking assessments at 4 mo corrected age and later follow up with the BSID III. The gestational age ranged from $22+2$ to $31+6 \mathrm{wk}$ and birth weight from 520 to $2,030 \mathrm{~g}$. Cranial ultrasound was performed at least twice in the neonatal period, at 2-7 d and at 35-36 gestational weeks, and all infants were regularly screened for retinopathy of prematurity. Bronchopulmonary dysplasia was defined as need for additional oxygen at 36 gestational weeks. Clinical routines have been described previously (13). The final study group did not differ from the initial LOVIS cohort neither in perinatal characteristics nor in the BSID III scores. The study was approved by the Regional Ethics committee at Uppsala University (Ups 03-665). Parental consent was collected for the clinical examinations and for the visual tracking assessments.

\section{Visual Tracking Assessments}

The child was placed securely in a baby seat inside a white cylinder ( $1 \mathrm{~m}$ diameter and $1 \mathrm{~m}$ high), and thereby shielded from distractors. A happy face $(7 \mathrm{~cm}$ diameter) with a camera at its nose moved horizontally according to a sinusoidal pattern at $0.25 \mathrm{~Hz}$ along a track on the inside of the cylinder surface (Figure 4). The camera made it possible to observe the child via a monitor, in order to ensure wellbeing and attention to the task. Two amplitudes of object motion were used, $12.5^{\circ}$ and $25^{\circ}$ of visual angle, and each trial lasted $35 \mathrm{~s}$.

Horizontal eye movements were recorded through electro-oculography (DatAid, Umeå, Sweden) (see refs. $(7,9)$ for detailed description). Miniature skin electrodes (Beckman, Grass technologies, West Warwick, RI) were placed at the outer canthi and the ground at the ear lobe or the forehead. A preamplifier was placed on the head of the infant, to minimize drift and noise. The electro-oculography was calibrated for each infant and the precision of eye position measurement was $0.4^{\circ}$.

Infants could move their heads rather freely during the trials. The movements of the head and the object were measured by an optoelectronic camera system (Qualysis Proreflex, Gothenburg, Sweden) utilizing infrared light and small (diameter $5 \mathrm{~mm}$ ) passive reflective markers. Measurement frequency was set to $240 \mathrm{~Hz}$ in synchrony with the electro-oculography. All infants tracked the object. In the rare case that an infant temporarily lost attention this section of tracking was not included in the analysis.

\section{Neurodevelopmental Assessment}

Neurodevelopmental follow up with the BSID III, performed at 29-41 (median 32) months' corrected age, was part of the standard clinical routine. The BSID III assesses the child's abilities within five subscales ( mean $=100, \mathrm{SD}=15)$ : cognition, receptive language, expressive language, fine motor, and gross motor functions. The cognitive subscale includes tasks such as shape perception, complexity of play behavior, simple visual logic, and comparisons. The receptive language subscale is based on identifying objects and actions in pictures, while the expressive language subscale is obtained through observation, 
naming of, and conversations around pictures. In the fine motor tasks, the child is asked to stack and string blocks and to handle a pencil and scissors. Stability, coordination, and balance are targeted in the gross motor tasks.

All children were tested by a licensed psychologist, who was blind to the results of the early visual tracking performance but could not, due to the clinical circumstances, be blind to gestational age.

\section{Ophthalmological Assessment}

Ophthalmologic and orthoptic assessment was performed at $30 \mathrm{mo}$ corrected age (33). In the present study, 64 of the 67 children were examined at $2.5 \mathrm{y}$, two at $6.5 \mathrm{y}$, and one at $1 \mathrm{y}$ corrected age. Four children had strabismus and eight had major refractive errors in at least one eye (defined as myopia of $<-3$ diopters hypermetropia $>+3$ diopters, astigmatismus $\geq 2$ diopters, and/or anisometropia $\geq 2$ diopters). Four of the latter children were wearing glasses at the examination. No child was visually impaired, i.e., blind or only able to fixate a torch when tested binocularly, regardless of degree of retinopathy of prematurity.

\section{Data Analysis}

Data from electro-oculography and Qualysis were synchronized to obtain data of head and eye movements in relation to the moving object. The analysis was performed by FYSTAT designed for time series (DatAid, Umeå, Sweden) or Matlab (Mathworks, Natick, MA). Gaze gain, smooth pursuit gain, head gain, and timing of gaze, were calculated from the average of the high and low amplitude trials $(7,9)$. Before the calculation of smooth pursuit, the saccades exceeding $40 \%$ were eliminated from the eye movement data (7).

Gaze gain and head gain were defined as their spatial position relative to that of the object. The smooth pursuit gain was defined as its spatial position to head slip, i.e., the difference between head and object positions or the proportion of the target trajectory that needs to be covered by the eye to obtain perfect gain.

Timing (phase) was calculated by cross correlations, where the time difference between gaze and object motion was estimated (Figure 2). Finally, the number of large saccades $\left(>100^{\circ} / \mathrm{s}\right)$ were counted from the eye velocity file.

\section{Statistical Analysis}

Statistical analysis was carried out with IBM SPSS Statistics, version 20.0. Armonk, NY, and graphs were created in GNU Octave 3.8.1. (Open source).

Similar to findings in the EXPRESS study (22), the BSID III language and motor subscales in this study differed in mean and SDs as compared to BSID III reference data (Table 1). Therefore, we used the results of each subscale in the analysis instead of language and motor indexes.

Associations between tracking parameters and BSID III results were analyzed through hierarchical regression models and effect size $\left(R^{2}\right)$ and significance levels ( $P$ values, two-tailed) were noted. Quadratic regression models were used for evaluating relationships between gaze gain and the BSID III subscales since both too low and too high gaze gain values means suboptimal tracking. All remaining parameters were analyzed with linear regression.

To test if adding gaze gain to a model of neonatal risk factors increased the capacity to predict neurodevelopmental outcome, a linear hierarchical regression analysis was conducted. To adjust for the nonlinear relationship between gaze gain and the BSID III sub scores, the gaze variable was squared. Gaze gain was then entered both as squared and nonsquared in step two of the hierarchical regression model. Following common praxis to include $1 / 10$ as many covariates as subjects, this meant that four neonatal risk factors could be entered into the first step of the hierarchical regression model. These risk factors were selected among factors of known relevance for neurodevelopment (34). Several of the neonatal risk factors were highly correlated with each other and collinearity diagnosis showed that birth weight and gestational age were too strongly interdependent to be entered into the same regression analysis. The degree of prematurity (gestational age) was selected as being more relevant for the study, along with bronchopulmonary dysplasia, retinopathy of prematurity, and severe brain injury (presence of intraventricular hemorrhage 3-4 and/or periventricular leukomalacia).

\section{ACKNOWLEDGMENTS}

We thank all children and parents who participated in the study; twentyone children also participated in the EXPRESS study. We acknowledge the work by the following: Jonina Hreinsdottir, Lisa Strömbeck-Sandlund, Cecilia Ewald, Uwe Ewald, Bo Strömberg, Olga Kuchokova, and Ingela Helling.

\section{STATEMENT OF FINANCIAL SUPPORT}

Financial support was obtained from the Linnéa and Josef Carlsson Foundation, Helsingborg, Sweden, the Jerring Foundation, Stockholm, Sweden, the Gillbergska Foundation, Uppsala, Sweden, and Uppsala University Funds, Uppsala, Sweden.

Disclosure: None of the authors have any conflict of interest or any financial ties to products in this study.

\section{REFERENCES}

1. Serenius F, Källén K, Blennow M, et al.; EXPRESS Group. Neurodevelopmental outcome in extremely preterm infants at 2.5 years after active perinatal care in Sweden. JAMA 2013;309:1810-20.

2. Aarnoudse-Moens CS, Weisglas-Kuperus N, van Goudoever JB, Oosterlaan J. Meta-analysis of neurobehavioral outcomes in very preterm and/or very low birth weight children. Pediatrics 2009;124:717-28.

3. Atkinson J, Braddick O. Visual and visuocognitive development in children born very prematurely. Prog Brain Res 2007;164:123-49.

4. Fazzi E, Bova S, Giovenzana A, Signorini S, Uggetti C, Bianchi P. Cognitive visual dysfunctions in preterm children with periventricular leukomalacia. Dev Med Child Neurol 2009;51:974-81.

5. Böhm B, Lundequist A, Smedler AC. Visual-motor and executive functions in children born preterm: The Bender Visual Motor Gestalt Test revisited. Scand J Psychol 2010;51:376-84.

6. Chau V, Synnes A, Grunau RE, Poskitt KJ, Brant R, Miller SP. Abnormal brain maturation in preterm neonates associated with adverse developmental outcomes. Neurology 2013;81:2082-9.

7. von Hofsten C, Rosander K. Development of smooth pursuit tracking in young infants. Vision Res 1997;37:1799-810.

8. Rosander K. Visual tracking and its relationship to cortical development. Prog Brain Res 2007;164:105-22.

9. Von Hofsten C, Rosander K. The development of gaze control and predictive tracking in young infants. Vision Res 1996;36:81-96.

10. Rommelse NN, Van der Stigchel S, Sergeant JA. A review on eye movement studies in childhood and adolescent psychiatry. Brain Cogn 2008;68: 391-414.

11. Luna B, Velanova K, Geier CF. Development of eye-movement control. Brain Cogn 2008;68:293-308.

12. Strand-Brodd K, Ewald U, Grönqvist H, et al. Development of smooth pursuit eye movements in very preterm infants: 1 . General aspects. Acta Paediatr 2011;100:983-91.

13. Brodd KS, Grönqvist H, Holmström G, Grönqvist E, Rosander K, Ewald U. Development of smooth pursuit eye movements in very preterm born infants: 3 . Association with perinatal risk factors. Acta Paediatr 2012;101:164-71.

14. Winges SA, Soechting JF. Spatial and temporal aspects of cognitive influences on smooth pursuit. Exp Brain Res 2011;211:27-36.

15. Koziol LF, Budding DE, Chidekel D. From movement to thought: executive function, embodied cognition, and the cerebellum. Cerebellum 2012;11:505-25.

16. Grönqvist H, Brodd KS, Rosander K. Development of smooth pursuit eye movements in very prematurely born infants: 2 . The low-risk subgroup. Acta Paediatr 2011;100:e5-11.

17. Jacobs M, Harris CM, Shawkat F, Taylor D. Smooth pursuit development in infants. Aust N Z J Ophthalmol 1997;25:199-206.

18. Atkinson J. The Developing Visual Brain. New York, NY: Oxford University Press Inc, 2000:28-42.

19. Ellis EM, Robledo Gonzalez M, Deák GO. Visual prediction in Infancy: What is the association with later vocabulary? Lang Learn Dev 2014;10:136-50.

20. von Hofsten C. Predictive reaching for moving objects by human infants. J Exp Child Psychol 1980;30:369-82. 


\section{Articles | Kauletal.}

21. von Hofsten C, Rönnqvist L. Preparation for grasping an object: a developmental study. J Exp Psychol Hum Percept Perform 1988;14:610-21.

22. Månsson J, Stjernqvist K. Children born extremely preterm show significant lower cognitive, language and motor function levels compared with children born at term, as measured by the Bayley-III at 2.5 years. Acta Paediatr 2014;103:504-11.

23. Ricci D, Romeo DM, Gallini F, et al. Early visual assessment in preterm infants with and without brain lesions: correlation with visual and neurodevelopmental outcome at 12 months. Early Hum Dev 2011;87: 177-82.

24. Stjerna S, Sairanen V, Gröhn R, et al. Visual fixation in human newborns correlates with extensive white matter networks and predicts long-term neurocognitive development. J Neurosci 2015;35:4824-9.

25. Von Hofsten C. Action, the foundation for cognitive development. Scand J Psychol 2009;50:617-23.

26. Wattam-Bell J, Birtles D, Nyström P, et al. Reorganization of global form and motion processing during human visual development. Curr Biol 2010;20:411-5.

27. Mercuri E, Atkinson J, Braddick $\mathrm{O}$, et al. Basal ganglia damage and impaired visual function in the newborn infant. Arch Dis Child Fetal Neonatal Ed 1997;77:F111-4.

28. Weinstein JM, Gilmore RO, Shaikh SM, et al. Defective motion processing in children with cerebral visual impairment due to periventricular white matter damage. Dev Med Child Neurol 2012;54:e1-8.
29. Shah DK, Guinane C, August P, et al. Reduced occipital regional volumes at term predict impaired visual function in early childhood in very low birth weight infants. Invest Ophthalmol Vis Sci 2006;47:3366-73.

30. Langaas T, Mon-Williams M, Wann JP, Pascal E, Thompson C. Eye movements, prematurity and developmental co-ordination disorder. Vision Res 1998; 38:1817-26.

31. Ment LR, Bada HS, Barnes P, et al. Practice parameter: neuroimaging of the neonate: report of the Quality Standards Subcommittee of the American Academy of Neurology and the Practice Committee of the Child Neurology Society. Neurology 2002;58:1726-38.

32. Hintz SR, Barnes PD, Bulas D, et al.; SUPPORT Study Group of the Eunice Kennedy Shriver National Institute of Child Health and Human Development Neonatal Research Network. Neuroimaging and neurodevelopmental outcome in extremely preterm infants. Pediatrics 2015;135:e32-42.

33. Hreinsdottir J, Ewald U, Strand Brodd K, Örnkloo H, von Hofsten C, Holmström G. Ophthalmological outcome and visuospatial ability in very preterm children measured at 2.5 years corrected age. Acta Paediatr 2013;102:1144-9.

34. Schmidt B, Asztalos EV, Roberts RS, Robertson CM, Sauve RS, Whitfield MF; Trial of Indomethacin Prophylaxis in Preterms (TIPP) Investigators. Impact of bronchopulmonary dysplasia, brain injury, and severe retinopathy on the outcome of extremely low-birth-weight infants at 18 months: results from the trial of indomethacin prophylaxis in preterms. JAMA 2003;289:1124-9. 